\title{
La Red Social Busuu en la Producción Oral en Francés
}

\section{The Busuu social network in French speaking skills}

\author{
Adrián Calderín Gutiérrez ${ }^{1}$ \\ https://orcid.org/0000-0002-4959-6162 \\ Universidad Tecnológica Indoamérica, Ecuador
}

Recibido: $10-05-2021$

Aceptado: 30-08-2021

\section{Cita Recomendada}

Calderín, A. (2021). La red social Busuu en la producción oral en Francés. Hamut'ay, 8 (2), 58-68 http://dx.doi.org/10.21503/hamu.v8i2.2293

\section{Resumen}

Esta investigación tuvo por objetivo implementar una metodología basada en la red especializada en idiomas Busuu para mejorar la producción oral en francés. Se llevó a cabo una investigación de campo, descriptiva con enfoque cuantitativo. Para ello, se creó un curso virtual con una versión gratuita de esta aplicación. La muestra final estuvo conformada por 63 personas de varios países de América Latina, miembros del Club de Francés-Amigos, quienes completaron todas las actividades propuestas en el curso. Los resultados mostraron que el 22\% de los participantes obtuvo una calificación de 20/20 en el examen final; el 17\% obtuvo calificaciones de 16/20; seguidos por los de 17 , con $16 \%, 18$ y 15 con un $13 \%$, respectivamente. Un $5 \%$ obtuvo 14 puntos, otro $5 \%, 10$, y el 2\%, 13 puntos. Los participantes que utilizaron Busuu entre una y dos o más de dos horas semanales, manifestaron un elevado nivel de satisfacción, y se concluyó que esta metodología resultó ser muy efectiva para ellos; lo que evidencia la eficacia de las redes sociales para potenciar el aprendizaje de nuevas lenguas, sobre todo las competencias orales.

Palabras Clave: Busuu, expresión oral, francés, redes sociales, TIC.

\footnotetext{
1. Magister en Educación mención Pedagogía en Entornos Digitales. Liceo Franco- Ecuatoriano La Condamine, Quito, Ecuador; Licenciado en Lengua Francesa con Segunda Lengua Inglés. Email: adriancalderin080@gmail.com
} 


\begin{abstract}
This research aimed to implement a methodology based on the specialized network in Busuu languages to improve oral production in French. A descriptive field research with a quantitative approach was developed. For this, a virtual course was created with a free version of this application. The final sample consisted of 63 people from various Latin American countries, members of the Club de Frances-Amigos, who completed all the activities proposed in the course. The results showed that $22 \%$ of the participants scored $20 / 20$ on the final exam; $17 \%$ scored 16/20; followed by those of 17 , with $16 \%, 18$ and 15 with $13 \%$, respectively. $5 \%$ obtained 14 points, another 5\%, 10, and 2\%, 13 points. Participants who used Busuu between one and two or more than two hours per week, expressed a high level of satisfaction, therefore, it was concluded that this methodology proved to be very effective for them, which evidences the effectiveness of social networks to enhance the learning of new languages, especially oral skills.
\end{abstract}

Keywords: Busuu, oral expression, French, social networks, ICT.

\section{Introducción}

En la era digital que se vive, han proliferado y evolucionado múltiples herramientas que se encuentran en constante estado de cambio (Read y Barcena, 2020); las cuales han sido incorporadas por las nuevas generaciones en su vida diaria, pese a notables desigualdades existentes (Granado, 2019); hecho al que no es ajeno el ámbito de la educación, dado que tanto en la escuela y en el desarrollo de la enseñanza y el aprendizaje, como en la vida laboral, se han visto favorecidas por la inserción de las Tecnologías de la Información y la Comunicación (TIC) (Rahmi, Fitriati, y Fachraini, 2019). Villafuerte y Romero, (2017) señalan que una actitud positiva contribuye a aumentar las posibilidades de éxito de los alumnos cuando utilizan dispositivos informáticos en sus diversos trabajos académicos. Dentro de este contexto, Kannan y Munday, (2018) afirman que en las últimas décadas, la integración y las aplicaciones de las TIC han modelado la manera de enseñar y aprender idiomas. Es así que, el campo de las lenguas extranjeras se ha visto altamente beneficiado por la aparición de las TIC (Rivera, 2013; Alkamel y Chouthaiwale, 2018). Aplicaciones y sitios web como Duolingo, Busuu, Memrise, Tandem, entre otras tienen altos niveles de popularidad, especialmente para el estudio del inglés, además de permitir mejorar ciertas habilidades, entre ellas el trabajo colaborativo de los estudiantes (Gil, 2019).

Frente al panorama hasta aquí descrito, este trabajo se propuso como objetivo implementar una metodología para mejorar la expresión oral y la pronunciación en los estudiantes de un curso virtual de francés mediante el uso de la red social especializada en idiomas Busuu. Para la realización del curso se contó con la participación de integrantes del Club de Francés-Amigos, un grupo de Facebook, conformado por personas en su mayoría de América Latina.

Las problemáticas fundamentales que se plantean en este trabajo son las deficiencias en la producción oral en idioma francés y el desconocimiento de ciertas herramientas tecnológicas para el aprendizaje de esta lengua. Según Lopez-Sintas, Lamberti y Jakkapong (2020), diversos factores como la edad, las desigualdades sociales, el estado civil y el nivel de educación influyen directamente en cada usuario de Internet, lo que se manifiesta ampliamente en América Latina. A esto hay que sumar cierta resistencia a la aplicación de métodos innovadores en la educación, la profunda brecha digital y deficientes políticas económicas. Se añade la escasa alfabetización digital dentro del profesorado, cuya actitud es vital para poner en práctica el uso de nuevas tecnologías (Akay, 2020). 
Algunos estudios que fundamentan la presente investigación son las de Vesselinov et al., (2021), ya que utilizaron técnicas e instrumentos muy similares aunque para el idioma español. Tomé (2020) abordó el uso de las TIC para perfeccionar la producción oral en francés, proceso en el que analizó el papel de blogs, podcasts, redes sociales, etc., en el desarrollo de la expresión oral y la pronunciación. En otro estudio, Citrayasa, (2019) describe e interpreta experiencias de un grupo de alumnos que utilizaron Busuu, en las que se desarrolló una gran preferencia por esta red social.

El impacto de las TIC en el apredizaje de lenguas

Vesselinov et al, (2021) en su estudio "The Busuu Efficacy Study", tomó una muestra aleatoria de 114 usuarios de Busuu, de los cuales un $35 \%$ eran principiantes (situados en el primer semestre de español) y un 65\% intermedios (semestre 2, 3, 4). Los participantes realizaron al principio del estudio dos pruebas lingüísticas: una de lectura/gramática (WebCAPE) y otra de competencia oral (TNT); luego utilizaron Busuu para estudiar español durante 2 meses y volvieron a realizar las mismas pruebas al final del estudio. Como resultados se obtuvieron que el $100 \%$ de los usuarios principiantes mejoraron en al menos una de las áreas: lectura/gramática o competencia oral, frente al $82 \%$ de los usuarios intermedios. El 75\% de los usuarios principiantes mejoraron su oralidad, frente al $68 \%$ de los usuarios intermedios. Y el $67 \%$ de los usuarios principiantes mejoraron tanto su gramática como su competencia oral, en comparación con el 50\% de los usuarios intermedios.

Tomé, (2020) realizó un estudio titulado "Developing speaking skills and learning pronunciation with new technologies in the French as a Foreign Language classroom", en el que se plantearon proyectos de telecolaboración para el desarrollo de competencias de expresión oral y pronunciación, en la que participaron estudiantes de la Universidad de León con un nivel A1 (según el marco Común Europeo de Referencia para las lenguas), los cuales fueron tutoreados por estudiantes franceses bajo la supervisión de un profesor. Se utilizó además la plataforma Moodle. Se determinó, al final del estudio, que el refuerzo, la autocorrección y el calentamiento vocal fueron los métodos más eficaces para resolver los problemas de pronunciación, así como el acompañamiento de un tutor para ayudar a corregir. Se concluyó que la aplicación de las TIC fue muy útil y que la mayoría de los estudiantes reaccionaron de manera positiva (Tomé, 2020).

Citrayasa, (2019), en su estudio "Junior High School students' lived experiences of learning English using Busuu" (Experiencias de aprendizaje de inglés utilizando Busuu en estudiantes de secundaria), observó que el aprendizaje ubicuo supuso una gran motivación para los participantes, algunos de los cuales manifestaron su preferencia por Busuu antes que por los libros de texto. A través de las actividades que la aplicación ofrece, la mayoría de los estudiantes pudo elevar su nivel de comprensión de la lengua.

Kuşçu, (2019) señala que aplicaciones como Duolingo y Memrise están diseñadas para trabajar de acuerdo con la teoría que favorece la enseñanza de un idioma a partir de palabras y frases que están diseñadas para aprender a utilizar la gramática en contexto, reforzadas con materiales auténticos diseñados por especialistas del área.

\section{Las redes sociales y los idiomas}

La adquisición de idiomas ha cambiado gracias a las innovaciones tecnológicas, las cuales ponen en manos de los estudiantes una variedad de materiales y recursos muy útiles para este proceso (Tomé, 2020). Teléfonos inteligentes, tablets y computadores, ofrecen la posibilidad a quienes aprenden una nueva lengua, la oportunidad de poder estudiar en cualquier lugar y momento. Ramírez, Casillas y Contreras, (2014) enumeran varias herramientas digitales para aprender una nueva lengua y señala la importancia de las TIC en la modernización de este campo, aspecto que se fortalecen si se cuenta con Internet más 
accesible para la educación (Blank y Lutz, 2018). En el siglo XXI, se reconoce cada vez más que Internet (Sánvicen y Molina, 2015) y las redes sociales brindan acceso a un aprendizaje informal continuo a partir de múltiples experiencias en diversas áreas de conocimiento, que complementan lo adquirido con el aprendizaje formal (Chik y Ho, 2017). A su vez, es cada vez más común acceder a los recursos en los móviles como puente entre distintos entornos (Zourou, 2019).

Debido al hecho de que la lengua es intrínsecamente social, las redes sociales permiten una interacción muy positiva en la adquisición de los idiomas. Según Cervantes, (2019) las redes sociales se han vuelto un elemento indispensable en el aprendizaje de lenguas extranjeras. Para Abdelouahed (2019), la adecuada combinación del e-learning con la enseñanza tradicional puede aportar numerosos beneficios en el proceso de enseñanza y aprendizaje de una nueva lengua.

Wong, Sing y Aw (2017) proponen que las redes sociales pueden ser utilizadas para la promoción del aprendizaje contextualizado y conectado y las interacciones a través de aspectos de la vida cotidiana. Esto generaría usuarios más activos y más autónomos, con una menor necesidad de la participación de un profesor.

Aunque aplicaciones como Busuu, Babbel, Duolingo, Memrise, ofrecen la posibilidad del aprendizaje ubicuo, hay que señalar que la mayoría no han sido desarrolladas por expertos en campo de la pedagogía y que su eficacia puede ser puesta en duda (Nushi y Jenabzadeh, 2016). A continuación, se puede ver una breve enumeración de las redes más importantes en lo que respecta al campo de los idiomas:

1. Busuu. Cuenta con unos 115 millones de usuarios registrados en alrededor de 190 países y tiene características muy similares a Facebook, como las solicitudes de amistad, los grupos y los chats (Vesselinov et al., 2021).

2. Italki. Es una red social que conecta a estudiantes y profesores de idiomas a través del videochat. El sitio permite a los estudiantes encontrar profesores en línea para dar clases particulares, y a los profesores ganar dinero como tutores autónomos (Turula, 2017)

3. Memrise. Es una plataforma lingüística que utiliza la repetición espaciada de tarjetas para aumentar el ritmo de aprendizaje. Ofrece contenidos generados por los usuarios sobre una amplia gama de otras materias. La app de Memrise tiene cursos en 16 idiomas y sus combinaciones, mientras que la página web tiene disponibles muchos más idiomas. También se puede aprender inglés para usos específicos como negocios (Aminatun y Oktaviani, 2019)

4. Babbel. En su vertiente de red social, cuenta con un foro donde se puede chatear con miles de usuarios en el idioma que se elija (Nushi y Eqbali, 2018).

5. Skype. Conocida mundialmente por ser una prestigiosa herramienta on-line para realizar videollamadas, también ofrece la oportunidad de participar en la comunidad especialmente diseñada para el aprendizaje de idiomas (Austin, Hampel, y Kukulska-Hulme, 2017).

\section{Principales características de la red social Busuu}

Busuu es una aplicación con cerca de 115 millones de usuarios que ofrece lecciones de vocabulario y gramática a través de ejercicios concisos. Además, personas nativas pueden hacer comentarios escritos para corregir, lo que le otorga un aspecto social. Su lanzamiento se realizó en 2008 con 12 idiomas, organizada en diferentes lecciones y temáticas (Winans, 2020). Aunque muchas de sus funciones solo están disponibles para el servicio Premium, la versión gratuita a través de tarjetas de memoria permite aprender un idioma y realizar un número limitado de actividades de aprendizaje de vocabulario y de comprensión oral (Shibbata, 2020). Hasta el momento, el nivel de competencia más alto es el B2 del Marco Común Europeo de Referencia para las lenguas (CEFR por sus siglas en inglés), lo que corresponde a un usuario que es capaz de comprender las ideas tanto abstractas como concretas de un texto complejo.

Como en cualquier red social, está disponible la función de sugerencias de amigos, basadas en los idiomas que se estén estudiando. De esa 
manera pueden recibirse y ofrecerse correcciones de nativos. Cuenta además, con lecciones secuenciales con los tradicionales ejercicios de arrastrar y soltar, selección de palabras y rellenar espacios, entre otros (Winans, 2020). El sistema proporciona estadísticas del avance de cada usuario (palabras aprendidas, número de correcciones y votos para la mejor corrección). Por el momento, existe la limitación de contactar amigos por un chat directo. Su aspecto social es lo que hace más atractiva a esta aplicación. También puede decirse que se trata de una plataforma que trabaja con la traducción para propiciar la enseñanza de idiomas (Nunes et al., 2017).

Existe además, la actividad llamada "quiz", que contiene ejercicios de selección múltiple y llenar espacios. Se pueden organizar frases y escribir las palabras escuchadas en un audio, para posteriormente encontrar un sinónimo de la misma. Sin embargo, aunque los usuarios con planes de suscripción pueden realizar evaluaciones de escritura y de expresión oral sobre los temas objetivo, todas las tareas consisten en preguntas de respuesta corta y no se ofrecen oportunidades para negociar el significado. Por lo tanto, las tareas de evaluación del curso no tienen una gran autenticidad o interactividad (Shibbata, 2020).

\section{Busuu en el aprendizaje del francés}

Quinaux, (2017) examinó aplicaciones como ITooch, Busuu y Wallangues con el objetivo de disponer de herramientas fiables que pudieran ser utilizadas directamente en cursos de formación, en instituciones sin fines de lucro o en institutos con recursos limitados y, por otro lado, cuestionar la contribución real de estas para los hablantes no nativos. En este estudio se compararon las aplicaciones mencionadas y se enumeraron sus pros y sus contras. En el caso de Busuu, se asevera que existen errores ortográficos y no siempre las imágenes ayudan al estudiante a guiarse. Además, se critica su espíritu mercantil, ya que no se pueden realizar algunas actividades si no se paga la versión Premium (Shibbata, 2020).
En una investigación realizada por Padang, Pramuniati y Ratna, (2020) se profundiza en los errores gramaticales cometidos por los estudiantes que utilizaban Busuu para aprender francés, entre los que se encontró el modo del verbo con error más frecuente.

Potolia y Zourou, (2019) argumentan que herramientas como Busuu permiten que los estudiantes se familiaricen con prácticas de aprendizaje menos controladas y menos predefinidas que las del aula de idiomas, ya que el acompañamiento, función principal de un profesor de idiomas, puede ser ejercido conjuntamente por otros usuarios. Asi mismo, observaron que a veces no existe el rigor debido en las correcciones (cualquier usuario puede opinar sobre una producción), lo cual, genera a menudo tensiones en cuanto a la legitimidad del acto de corregir, así como un cierto desánimo ligado al carácter impersonal y sin seguimiento de la corrección.

Finalmente, la evaluación realizada en diversas investigaciones sobre la efectividad de Busuu como herramienta digital que propicia el aprendizaje de idiomas, muestra su importancia como plataforma emergente y sienta las bases para futuros estudios, sobre todo, en lo que concierne a la expresión oral, tal como se enfatiza en la presente investigación.

\section{Materiales y métodos}

Participantes

Para Arias, (2012) la muestra de un estudio comprende "el subconjunto, finito que puede dar cuenta de las características de todos los elementos" (p. 81). En este caso, el muestreo fue probabilístico, de tipo aleatorio estratificado (Hernández y Carpio, 2019), ya que se seleccionaron 63 estudiantes de varios países latinoamericanos que cumplieron con el requisito de completar todas las actividades del curso (Tabla 1). Para Hernández, Fernández y Baptista, (2016) la población de una investigación comprende "la totalidad del universo objetivo de estudio" (p.78), por lo que todos los integrantes del Club de Francés - Amigos conformaron la población 
en este caso. En el momento del estudio el grupo contaba con aproximadamente 20000 miembros a los cuales se lanzó la convocatoria del curso (Tabla 2).

Tabla 1. Muestra.

\begin{tabular}{ccc}
\hline \multicolumn{3}{c}{ UNIDADES DE OBSERVACION } \\
\hline ESTUDIANTES & 63 & $100 \%$ \\
\hline TOTAL & 63 & $100 \%$ \\
\hline
\end{tabular}

Fuente: Elaboración propia (2021).

Tabla 2. Población.

\begin{tabular}{ccc}
\hline \multicolumn{3}{c}{ UNIDADES DE OBSERVACION } \\
\hline DOCENTES & 1 & 0,00005 \\
ESTUDIANTES & 20.000 & 0,99999 \\
\hline TOTAL & 20.001 & 100 \\
Fuente: Elaboración propia & $(2021)$.
\end{tabular}

\section{Instrumento}

El instrumento utilizado para conocer el nivel alcanzado en la producción oral por los participantes en este curso fue la rúbrica oficial del CIEP (Centre International d'Études Pédagogiques), la cual se utiliza en todo el mundo en las evaluaciones de francés por instituciones como la Alianza Francesa. Este instrumento fue validado por tres expertos en el campo de la enseñanza de las lenguas extranjeras. La confiabilidad del mismo está respaldada por el propio CIEP y por DELF-DALF, instituciones que eminten los certificados internacionales de adquisición de la lengua francesa.

\section{Tipo y Diseño}

De acuerdo con Hernández, Fernández y Baptista, (2014) esta es una investigación descriptiva, ya que se realizan las observaciones y mediciones sin manipular variables. Se centró en recopilar datos e información (Campos, 2017). En consecuencia, la información obtenida permitió la descripción de la evolución y características de las actividades realizadas. Se trata, además, de una investigación básica, la cual posibilita la profundización de los conocimientos en un área determinada, sin contrastarlos con ningún aspecto práctico
(Muntané, 2010).

El enfoque es de corte cuantitativo, según Abero et al., (2015), las investigaciones cuantitativas se caracterizan por la explicación y descripción de la realidad y la generalización. En este estudio se realizaron dos encuestas para recolectar datos, una al inicio y una al final, así como un examen oral, el cual fue evaluado según la rúbrica de la Alianza Francesa. Además, cada semana hubo exámenes autoevaluados en la plataforma Canvas LMS.

\section{Procedimiento}

Para la realización de este estudio se procedió de la siguiente forma (Tabla 3):

Tabla 3. Etapas del procedimiento del estudio

\begin{tabular}{cl}
\hline ETAPA & \multicolumn{1}{c}{ DESCRIPCIÓN } \\
\hline $\mathbf{1}$ & $\begin{array}{l}\text { Se llevó a cabo una revisión bibliográfica con el fin de establecer los objetivos y } \\
\text { seleccionar un instrumento de medición adecuado. }\end{array}$ \\
\hline $\mathbf{2}$ & Se hizo una convocatoria en el Club de Francés Amigos para obtener los participantes. \\
\hline $\mathbf{3}$ & $\begin{array}{l}\text { Se realizaron reuniones vía Zoom para explicar a los interesados el funcionamiento del } \\
\text { curso y las herramientas a utilizar. }\end{array}$ \\
\hline $\mathbf{4}$ & $\begin{array}{l}\text { Se implementó un curso de cuatro semanas en la plataforma CANVAS LMS con } \\
\text { evaluaciones semanales y se realizó una prueba oral final, la cual fue calificada con ayuda } \\
\text { de la rúbrica utilizada en las certificaciones internacionales de francés. Este instrumento } \\
\text { fue validado por expertos, en este caso, profesores cuyo nivel de francés está acreditado } \\
\text { por la Alianza Francesa. }\end{array}$ \\
\hline $\mathbf{5}$ & Se recolectaron datos informativos a través de una encuesta de Google Forms. \\
\hline
\end{tabular}

5 Se recolectaron datos informativos a través de una encuesta de Google Forms.

Fuente: Elaboración propia (2021).

Confidencialidad y Consentimiento informado.

Los participantes en este estudio se inscribieron de manera voluntaria. Como estudiantes del Club de Francés Amigos, interesados en conocer su nivel de francés y desarrollarlo, se les informó que los datos recolectados se utilizarían en la investigación y estuvieron de acuerdo en que se les aplicaran los instrumentos mencionados para conocer su nivel en el idioma. Se les garantizó a los participantes que toda la información sería tratada de manera confidencial.

\section{Resultados}

En la Tabla 4, se presenta la distribución de participantes por países. 
Tabla 4. Participantes por países

\begin{tabular}{ccc} 
País & Personas & Porcentaje \\
\hline México & 21 & $33.3 \%$ \\
\hline Argentina & 10 & $15.8 \%$ \\
\hline Colombia & 10 & $15.8 \%$ \\
\hline Perú & 6 & $9.5 \%$ \\
\hline Ecuador & 5 & $7.9 \%$ \\
\hline Venezuela & 4 & $6.3 \%$ \\
\hline Guatemala & 3 & $4.8 \%$ \\
\hline Otros & 4 & $6.3 \%$ \\
\hline \multicolumn{2}{c}{ Fuente: Elaboración propia (2021). }
\end{tabular}

Méxicoaportóla mayorcantidadderepresentantes a la muestra, con un 33,3\%, seguido de Argentina y Colombia con un $15,8 \%$ cada uno; Perú aportó un $9.5 \%$; Ecuador un $7.9 \%$; Venezuela un 6,3\%, y Guatemala un $4.8 \%$. Entre personas de otros países como Chile, Bolivia, Honduras, Costa Rica apenas alcanzan el 6.3\%. A continuación, se presentan los resultados obtenidos durante el proceso investigativo.

En la Figura 1 se observa que el $90.5 \%$ de los participantes en el examen oral A1.1, fueron mujeres y el 9,5\% hombres, lo que arrojó que las mujeres mostraron más interés por este tipo de cursos.

Sexo

63 respuestas

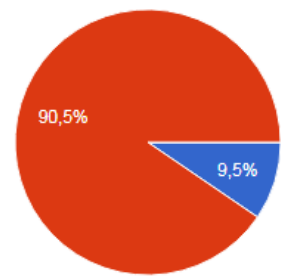

Masculino

- Femenino - Otro

Figura 1 Resultados de la encuesta final. Sexo de los participantes Fuente: Elaboración propia (2021).

En la Figura 2 se observa que el 28,6\% de los participantes en el examen fueron personas de 25 a 35 años; el segundo lugar fue ocupado por personas de 46 años en adelante, con un 27\%; luego los de 18 a 25 y de 36 a 45, ambos con $22,2 \%$ cada grupo.
¿En qué grupo etario se encuentra?

63 respuestas

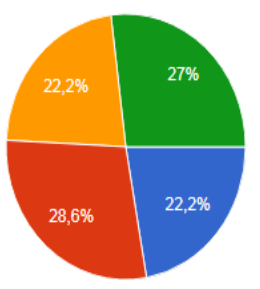

18 a 25 años

25 a 35 años

46 años en adelante

Figura 2 Grupo etario

Fuente: Elaboración propia (2021).

En lo referente al nivel de estudios de los participantes (Figura 3), se advirtió que las personas con educación universitaria concluida conformaron el grupo más significativo, con $41,3 \%$; en segundo lugar se ubicaron quienes culminaron el bachillerato, con un 20,6\%, y a continuación las personas con postgrado en un 17,5\%; en la última posición se encontraron aquellos que aún cursaban la universidad, representados por el 15,9\%.

¿Cuál es su nivel de estudios?

63 respuestas

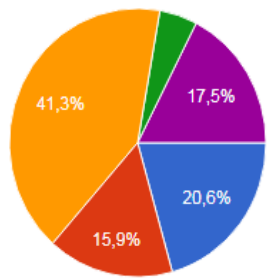

Bachillerato (finalizado)

Universidad (en curso)

Universidad (finalizada) Posgrado (en curso)

Posgrado (finalizado)

Figura 3 Nivel de estudios Fuente: Elaboración propia (2021).

Al inicio del curso, la mayor parte de participantes, es decir, alrededor del 50\% manifestó tener un nivel mínimo (Figura 4). Los demás manifestaron tener niveles ligeramente más altos, pero no significativamente si nos basamos en el MCER. Menos del 10\% declaró tener un nivel relativamente alto. La mayoría de los inscritos en el curso presentaron un nivel mínimo de francés para el momento del inicio de las actividades, 
Con qué nivel de francés comenzó este curso en una escala del 1 al 10, siendo 1 la calificación más baja y 10 la más alta?

63 respuestas

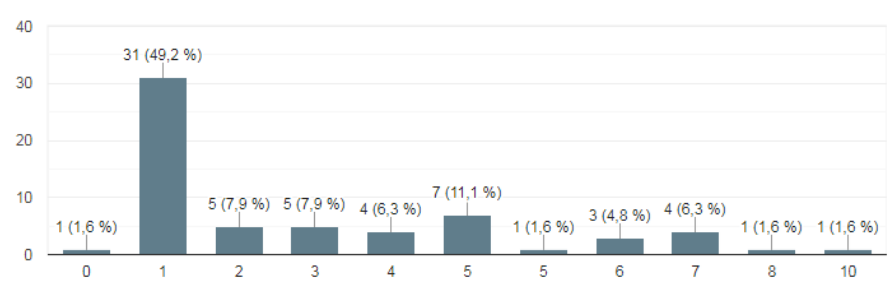

Figura 4. Nivel de francés para el inicio del curso Fuente: Elaboración propia (2021).

En la Figura 5 se observa que el $52,4 \%$ de los participantes empleó Busuu de una a dos horas; $31,7 \%$ más de dos horas y el $15,9 \%$ menos de una hora. La mayor parte de los participantes ocuparon la aplicación de una a dos horas por semana, seguidos de quienes la emplearon más de dos horas.

¿Cuántas horas utilizó la red social Busuu como promedio por semana? 63 respuestas

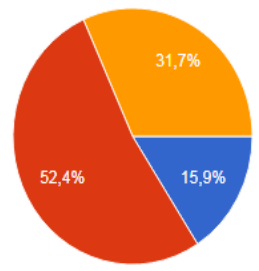

Figura 5. Horas de utilización de la red social Busuu. Fuente: Elaboración propia (2021).

En la Figura 6 se observa que el nivel de satisfacción alcanzó el $92.1 \%$, aunque un porcentaje menor manifestó su insatisfacción o su neutralidad.

¿Cómo valora su nivel de satisfacción con este curso?

63 respuestas

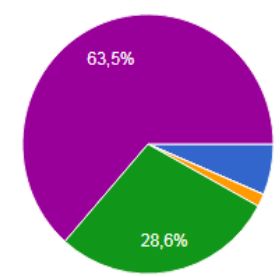

- Muy insatisfecho

- Insatisfech

Neutral

Satisfecho

- Muy satisfecho

Figura 6. Nivel de satisfacción con el curso. Fuente: Elaboración propia (2021).

En la Figura 7 se observa que el $22 \%$ de los participantes obtuvo una calificación de 20/20 en el examen final; el $17 \%$ obtuvo calificaciones de 16/20; seguidos por los de 17, con 16\%, 18 y 15 con un $13 \%$, respectivamente. Un 5\% obtuvo 14 puntos, otro $5 \%, 10$, y el $2 \%, 13$ puntos.

Resultados de la prueba oral final

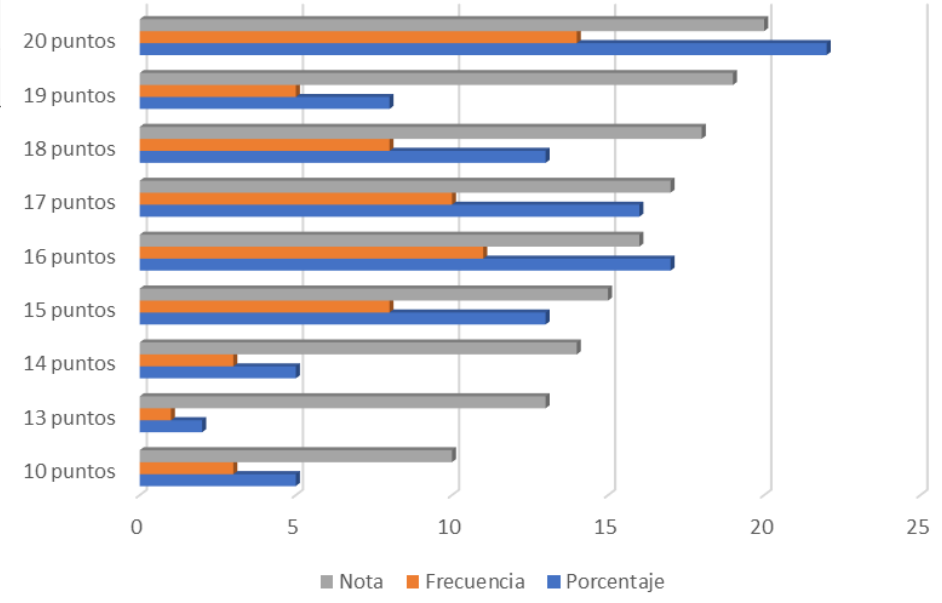

Figura 7. Resultados de la prueba oral final Fuente: Elaboración propia (2021).

\section{Discusión y conclusiones}

Este estudio reafirmó la tendencia tradicional del sexo femenino por las humanidades, en este caso el estudio de lenguas, ya que el $90.5 \%$ de los participantes fueron mujeres. Además, las personas activas en el ámbito académico (las cuales representaron el $79.4 \%$ de la muestra) tuvieron una mayor disposición para finalizar el curso y presentar el examen final del mismo. Esto se debe a que poseen una mayor disciplina, además de la exposición al estudio de alguna lengua extranjera, sobre todo los que cursan la universidad. Las diversas nacionalidades que participaron en el estudio reflejan el interés de países de la región por el aprendizaje del francés. Los participantes de 20 a 35 años y los mayores de 46 fueron quienes mayoritariamente finalizaron todas las actividades del curso y el examen oral A1.1, lo que revelaría que en estas edades se manifestó mayor interés por el aprendizaje del idioma y además una mayor predisposición por culminar el curso virtual. Esto es el resultado de una fuerte motivación a la hora de aprender una lengua extranjera.

En cuanto a los resultados de la prueba oral, el $93 \%$ de las personas calificaciones por encima 
de 14. Con una nota promedio de 17,03, o sea, un promedio general alto, teniendo en cuenta que 20 era la nota más alta. Esto muestra la eficacia de Busuu como alternativa a los cursos tradicionales. Como en el trabajo desarrollado por Tomé, (2020) los alumnos experimentaron grandes avances en la producción oral, sobre todo aquellos que manifestaron haber comenzado el curso con un nivel básico. En general, la cantidad de horas destinadas al uso de esta aplicación se corresponde con lo descrito en otras investigaciones como la realizada por Vesselinov et al, (2021) donde se solicitó a los usuarios un tiempo de dos horas semanales.

Los altos niveles de satisfacción alcanzaron aproximadamente el $92 \%$ de los participantes, lo cual puede considerarse como muy bueno, ya que se localiza por encima del $50 \%$ de los participantes. Como en la investigación desarrollada por Citrayasa (2019), las personas hicieron de Busuu una de sus aplicaciones preferidas para estudiar idiomas.

Esta investigación logró potenciar una tecnología innovadora para fortalecer las destrezas orales en francés, e incluso la pronunciación de los involucrados en el estudio, por medio de Busuu. Para ello se diseñó un curso virtual de cuatro semanas en CANVAS LMS, el cual redirigía a las lecciones de Bussu. Gracias al compromiso y motivación de los participantes se alcanzó un alto nivel de satisfacción al término del curso.

Algunas de las ventajas destacables que se observaron tras utilizar la red social Busuu fueron que existe la posibilidad de interactuar con nativos, además constituye un punto de encuentro de personas de todo el planeta apasionadas por el aprendizaje de idiomas, como refuerzo del trabajo colaborativo $y$ la retroalimentación. Esta red propicia el aprendizaje ubicuo, ya que al tener una aplicación móvil, facilita el acceso desde cualquier lugar y en cualquier momento. En esta plataforma se pueden encontrar, aunque no de manera gratuita, los servicios de profesionales del idioma. La red es una poderosa herramienta para perfeccionar las competencias de expresión oral, sobre todo, gracias a todas sus funcionalidades.
Los estudios futuros se beneficiarían de investigar la eficacia en otros idiomas como el inglés, el portugués, el italiano o el alemán.

Para tener una mayor confianza en los resultados, los estudios futuros podrían utilizar un estudio comparativo que contrastara los resultados de una aplicación como Busuu con los resultados de un semestre universitario tradicional presencial de estudio de idiomas.

\section{Agradecimientos}

M.A Stalyn Ávila Herrera por su tutoría en la tesis de maestría en la Universidad Tecnológica Indoamérica que hizo posible la publicación de este artículo.

\section{Referencias Bibliográficas}

Abdelouahed, L. (2019). The use of e-learning in foreign language learning: A Case Study of Undergraduate EFL Students. International Journal of Language and Literary Studies, 1(3), 30-42. https://doi.org/10.36892/ijlls.v1i3.79

Abero, L., Berardi, L., Capocasale, A., y García, S. y. (2015). Investigación Educativa: Abriendo puertas al conocimiento. Quito: CLACSO.

Akay, C. (2020). In-Service Teachers' Resistance To Technology: A Qualitative Meta-Synthesis By Entreq Statement (1998-2018). IJETSAR (International Journal of Education Technology and Scientific Researches), 924-944. https://doi. org/10.35826/ijetsar.154

Alkamel, M. A. A., \& Chouthaiwale, S. S. (2018). The use of ICT tools in English language teaching and learning: A literature review. Veda's Journal of English Language and Literature, 5(2), 2933.

Aminatun, D., \& Oktaviani, L. (2019). Memrise: Promoting students' autonomous learning skill through language learning application. Metathesis: Journal of English Language, Literature, and Teaching, 3(2), 214-223. https:// doi.org/10.31002/metathesis.v3i2.1982

Arias, F. (2012). El proyecto de Investigación (Sexta ed.). Caracas: Episteme.

Austin, N., Hampel, R., \& Kukulska-Hulme, A. 
(2017). Video conferencing and multimodal expression of voice: Children's conversations using Skype for second language development in a telecollaborative setting. System, 64, 87-103. https://doi.org/10.1016/j.system.2016.12.003 Blank, G., \& Lutz, C. (2018). Benefits and harms from Internet use: A differentiated analysis of Great Britain. New media \& society, 20(2), 618640. https://doi.org/10.1177/1461444816667135 Campos, O. (2017). Métodos de Investigación Académica . Costa Rica: Universidad de Costa Rica.

Cervantes, D. (2019). Las redes sociales y el aprendizaje de la lengua extranjera. Revista. Boletín redipe, 8(11), 18-22.

https://doi.org/10.36260/rbr.v8i11.855

Chik, A., \& Ho, J. (2017). Learn a language for free: Recreational learning among adults. System, 69, 162-171. https://doi.org/10.1016/j. system.2017.07.017

Citrayasa, V. (2019). Junior High School students' lived experiences of learning English using Busuu. Indonesia EFL Journal, 5(2), 85-92.

https://doi.org/10.25134/ieflj.v5i2.1900

Gil, N. (2019). Ambiente virtual de aprendizaje: beneficios y ventajas para la enseñanza del francés como L2. Revista Boletín Redipe, 8(11), 91 - 99. https://doi.org/10.36260/rbr.v8i11.852

Granado P., M. (2019). Educación y exclusión digital: los falsos nativos digitales. Revista de estudios socioeducativos, RESED, (7), 27-41.

https://doi.org/10.25267/Rev_estud_ socioeducativos.2019.i7.02

Hernández S., R., Fernández C., C., y Baptista L., P. (2014). Metodología de la Investigación. Mexico : Mc Graw Hill-Education.

Hernández, C. E., y Carpio, N. (2019). Introducción a los tipos de muestreo. Alerta, Revista científica del Instituto Nacional de Salud, 2(1), 75-79.

https://doi.org/10.5377/alerta.v2i1.7535

Kannan, J., \& Munday, P. (2018). New Trends in Second Language Learning and Teaching through the lens of ICT, Networked Learning, and Artificial Intelligence. Círculo de Lingüística Aplicada a la Comunicación, (76), 13-30. https://doi.org/10.5209/CLAC.62495
Kuşçu, E. (2019). Applications for Mobile Assisted French Learning: Duolingo And Memrise. International Journal of Language Academy, 7(4), 304-318.

https://doi.org/10.29228/ijla.25833

Lopez-Sintas, J., Lamberti, G., \& Sukphan, J. (2020). The social structuring of the digital gap in a developing country. The impact of computer and internet access opportunities on internet use in Thailand. Technology in Society, 63, 101433. https://doi.org/10.1016/j.techsoc.2020.101433 Muntané R., J. (2010). Introducción a la investigación básica. RAPD ONLINE, 33(3), 221-227.

Nunes, G. M., Leffa, V. J., Lopes, J. A., y Oliveira, V. O. (2017). O uso do Feedback automático no aplicativo educacional Busuu e sua influência na aprendizagem de línguas. Revista Linguagem em Foco, 9(1), 25-38.

Nushi, M., \& Eqbali, M. H. (2018). Babbel: A mobile language learning app. TESL Reporter, 51(1), 109-121.

Nushi, M., \& Jenabzadeh, H. (2016). Busuu--The Mobile App. TESL Reporter, 49(2), 30-38.

Padang, S. P., Pramuniati, I. P., \& Soraya, T. R. Analyse des erreurs grammaticale des écritures chez les étudiants de la section française dans l'application de Busuu. HEXAGONE Jurnal Pendidikan, Linguistik, Budaya dan Sastra Perancis, 9(2), 832-840.

Potolia, A., y Zourou, K. (2019). Approches réflexives sur l'accompagnement pédagogique au sein d'une communauté web 2.0 d'apprenants de langues. Distances et médiations des savoirs(26). https://doi.org/10.4000/dms.3639

Quinaux, E. (2017). L'enseignement du FLE par les applications en ligne : une didactique novatrice? Documents pour l'histoire du français langue étrangère ou seconde, 58-59. https://doi. org/10.4000/dhfles.4457

Rahmi, R., Fitriati, F., \& Fachraini, S. (2019). An Analysis of Teachers' Perceptions toward the Role of ICT Based Media in Teaching and Learning Process among Primary Schools' Teachers. Jurnal Ilmiah Peuradeun, The InternationalJournal of Social Sciences, 7(3), 469-482. https://doi. org/10.26811/peuradeun.v7i3.335 
Ramírez M., A., Casillas A., M. Á., \& Contreras A., C. C. (2014). La incorporación de las TIC a la enseñanza universitaria de los idiomas. Debate Universitario CAEE-UAI, 3(5), 125-140.

Read, T., \& Barcena, E. (2020). Toward a framework for language MOOCs and mobile assisted language learning. Propósitos y Representaciones, 8(1), e470.

https://doi.org/10.20511/pyr2020.v8n1.470

Rivera, A. (2013). Impacto de los Ambientes Virtuales de Aprendizaje (AVA) en el desarrollo de competencias linguisticas en tareas comunicativas básicas del inglés, en alumnos de bachillerato (Tesis de maestría). Tecnológico de Monterrey, México, D.F., México.

Sánvicen, P., y Molina, F. (2015). Efectos del uso de Internet como fuente principal de información. Prisma Social, (15), 352 - 386.

Shibata, N. (2020). The Usefulness of Busuu Online Courses for Foreign Language Learning. Computer-Assisted Language Learning Electronic Journal, 21(2), 197-203.

Tomé, M. (2020). Developing speaking skills and learning pronunciation with new technologies in the French as a Foreign Language classroom. Archivum, 70(2), 325-358. https://doi. org/10.17811/arc.70.2.2020.325-358

Turula, A. (2017). Learner autonomy as a social construct in the context of Italki. Teaching English with Technology, 17(2), 3-28.

Vesselinov, R., Grego, J., Tasseva-Kurticheva, M., \& Sedaghatgoftar, N. (2021). The Busuu Efficacy Study. University of Maryland, University of South Carolina, University of South Carolina, Kharazmi University.

Villafuerte, J., \& Romero, A. (2017). Learners' Attitudes toward Foreign Language Practice on Social Network Sites. Journal of education and learning, 6(4), 145-158. https://doi.org/10.5539/ jel.v6n4p145

Winans, M. D. (2019). Busuu: A Social Network Application to Learn Languages. Calico Journal, 37(1), 117-126.

https://doi.org/10.1558/cj.37781

Wong, L. H., Chai, C. S., y Aw, G. P. (2017). Aprendizaje de idiomas «sin costuras»: Aprendizaje de segundas lenguas y redes sociales. Comunicar, 25(50), 9-20. https://doi. org/10.3916/C50-2017-01

Zourou, K. (2019). A Critical Review of Social Networks for Language Learning Beyond the Classroom. In M. Dressman, \& R. W. Sadler, The Handbook of Informal Language Learning (pp. 370-382).

https://doi.org/10.1002/9781119472384.ch24 